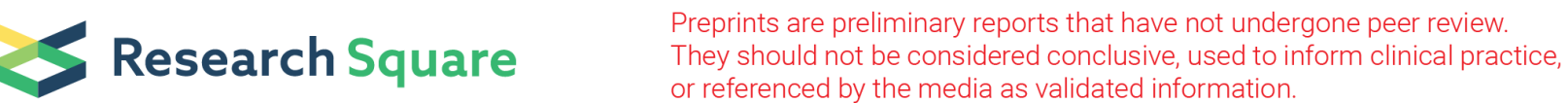

\section{A Combined Analysis of the Transcriptome and Metabolome Revealed the Molecular Mechanism by which GA3 Disrupts Dormancy in Leymus Chinensis Seeds}

\section{Bing Li}

Northeast Agricultural University

Pan Zhang

Northeast Agricultural University

Fengdan Wang

Northeast Agricultural University

Ran Li

Northeast Agricultural University

Jian Liu

Jilin Academy of Agricultural Sciences

Qiannan Wang

Northeast Agricultural University

Wei Liu

Northeast Agricultural University

\section{Bo Wang}

Northeast Agricultural University

Guofu Hu ( $\sim$ guofuh2003@163.com )

Northeast Agricultural University https://orcid.org/0000-0002-3630-4503

Research article

Keywords: Leymus chinensis, Seed dormancy, Transcriptome, Metabolome, GA3, Dormancy disruption

Posted Date: July 27th, 2020

DOI: https://doi.org/10.21203/rs.3.rs-41835/v1

License: (c) (1) This work is licensed under a Creative Commons Attribution 4.0 International License.

Read Full License 


\section{Abstract}

Background: Leymus chinensis is a perennial forage grass that has good palatability, high yield and high feed value, but seed dormancy is a major problem limiting the widespread cultivation of L. chinensis. The aim of this study was to investigate the underlying molecular mechanism and identify candidate genes associated with dormancy disruption by gibberellic acid $\left(\mathrm{GA}_{3}\right)$ through transcriptomic and metabolomic analysis.

Results: The germination test revealed that the optimum concentration of $\mathrm{GA}_{3}$ for disruption of $L$. chinensis seed dormancy was $200 \mu \mathrm{g} / \mathrm{L}$. Compared with seeds soaked in sterile water, a total of 4,327 and 11,919 differentially expressed genes (DEGs) and 871 and 650 differentially abundant metabolites were identified in de-hulled and hulled seeds treated with $\mathrm{GA}_{3}$, respectively. Most of the DEGs were associated with starch and sucrose metabolism, protein processing in the endoplasmic reticulum, endocytosis and ribosomes. Furthermore, isoquinoline alkaloid biosynthesis, tyrosine metabolism, starch and sucrose metabolism, arginine and proline metabolism, and amino sugar and nucleotide sugar metabolism were significantly enriched pathways. Integrative analysis of the transcriptomic and metabolomic data revealed that starch and sucrose metabolism is one of the most important pathways that may play a key role in the energy supply for the transition of $L$. chinensis seeds from a dormant state to germination by suppressing the expression of Cel61a, egID, cel1, tpSA, SPAC2E11.16c and TPP2, along with enhancing the expression of $A M Y 1.1, A M Y 1.2, A M Y 1.6$ and GLIP5, and finally inhibiting the synthesis of cellobiose, cellodextrin, and trehalose while promoting the hydrolysis of sucrose, starch, cellobiose, cellodextrin, and trehalose to glucose.

Conclusions: This study identified several key genes and provided new insights into the molecular mechanism of seed dormancy release by $\mathrm{GA}_{3}$ in L. chinensis. These putative genes will be valuable resources for improving the seed germination rate in future breeding studies.

\section{Background}

Seed dormancy, a property formed at the last stage of seed development, is an adaptation mechanism preventing seed germination in unfavourable environments or regulating when and where the seeds germinate [1-3]. This dormant state helps maintain seed vigour until germination. Although seed dormancy can prevent germination, which is advantageous for storage after harvest, this state is unfavourable for crop cultivation. Understanding the mechanisms of dormancy and the methods of dormancy disruption, as well as regulating the transition of crop seeds from dormancy to germination, is extremely important for agricultural production [4].

Dormancy and germination are two complex biological processes influenced by genetic and many environmental factors, such as endogenous hormones, light, moisture and temperature. In the hypothesis regarding hormonal balance, abscisic acid (ABA) and gibberellin (GA) are a pair of very important hormono molonuloc that nlav an occontial rolo in cood normination and dormancy, and there is Loading [MathJax]/jax/output/CommonHTML/fonts/TeX/fontdata.js 
antagonism between these molecules [5]. ABA induces the formation of seed dormancy during seed maturation and maintains this state, while GA promotes dormancy release and germination [6], and the interaction between $A B A$ and GA during the metabolism and signal transduction process determines the final status of dormancy and germination in the seed [7]. During the transition of seeds from a dormant state to germination, carbohydrate metabolism and plant hormone signal transduction pathways are activated [8]. Some related genes involved in biosynthesis, antagonism and signalling pathways of GA and $A B A$ have already been identified in many plants. ABI4 is a key transcription factor that can be directly or indirectly involved in the regulation of key genes in the ABA and GA biogenesis pathways leading to seed dormancy [9]. The transcription factor REVEILLE1 (RVE1) interacts with REPRESSOR OF GA-LIKE2 (RGL2) to regulate the dormancy and germination of Arabidopsis thaliana seeds by integrating light perception, GA metabolism and the associated signalling pathways [10]. In the process of GAmediated promotion of seed germination, GA3ox 1 and GA3ox2 are two key genes regulating GA synthesis [11], while GA2ox promotes GA degradation [12]. Transcriptomic analysis found that transcripts of the GID1 family related to the GA pathway were upregulated during the dormancy release stages in grape, while transcripts of the DELLA family were downregulated [13]. In addition to the genetic changes, metabolites were also correlated with seed germination. Exogenous $\mathrm{GA}_{4}$ may play an important role in dormancy disruption by changing the abundances of metabolites involved in galactose, glyoxylate, dicarboxylate and starch and sucrose metabolism [14]. Thus, the change from a dormant state to germination in seeds is a complex process regulated by genes and metabolites.

Leymus chinensis, also known as alkali grass, is a perennial rhizome grass of Leymus Hochst. and is an important forage and soil and water conservation plant. This grass has important economic and ecological value due to properties such as cold tolerance, drought tolerance, salt tolerance and trampling tolerance. In the natural environment, $L$. chinensis is dominated by asexual propagation due to the long seed dormancy period and low germination rate, which has greatly restricted its extensive application in artificial grassland construction and degraded grassland restoration. Some studies have suggested that the mechanically tied lemmas and seed coats prevent the infiltration of accelerators and the exudation of inhibitors, leading to seed dormancy of $L$. chinensis, meanwhile, the large amount of $A B A$ in lemmas and seed coats is also a factor inhibiting seed germination [15-17]. With the development of animal husbandry and the strengthening of ecological environment management, there is an increased demand for improved seed quantity and quality of $L$. chinensis $[18,19]$. Therefore, dormancy disruption and improvement of the germination rate of $L$. chinensis seeds has become a hot topic. Seeds treated with variable temperature can promote germination to a certain extent, and the transcriptomic data of $L$. chinensis seed germination at variable temperature showed that the genes related to seed germination were Chi1, CBF3, GA3OX, EXPB4 and SAIN1 [20]. Additionally, studies on a variety of plants showed that gibberellic acid $\left(\mathrm{GA}_{3}\right)$ can effectively disrupt the physiological dormancy of seeds [21-23]. Therefore, the application of $\mathrm{GA}_{3}$ to enhance seed germination has provided new opportunities for the production of $L$. chinensis. Although studies on the physiological mechanism by which $\mathrm{GA}_{3}$ increases the germination rate of $L$. chinensis seeds have been reported, little attention has been paid to the molecular mechanism 
In this study, the optimal concentration of $\mathrm{GA}_{3}$ was first selected from hulled and de-hulled seeds of $L$. chinensis. Moreover, to elucidate the regulatory networks by which $\mathrm{GA}_{3}$ disrupts seed dormancy and to explore some putative genes in $L$. chinensis, we conducted transcriptome sequencing and metabonomic analysis using seeds of $L$. chinensis soaked in $200 \mu \mathrm{g} / \mathrm{L} \mathrm{GA}_{3}$ for $24 \mathrm{~h}$. The results of this study will provide a theoretical basis for biological research on L. chinensis seeds and will be helpful for improving the germination rate in L. chinensis breeding.

\section{Results}

Effect of $\mathrm{GA}_{3}$ on the germination rate, germination index and germination potential of Leymus chinensis seeds

Three concentrations of $\mathrm{GA}_{3}$ significantly promoted germination of L. chinensis seeds, either hulled or dehulled, compared to the control group, and the hulls that covered the seeds inhibited germination, leading to a delayed initial germination time and decreased germination rate (Fig. 1). The initial germination time of de-hulled seeds was day 2, while hulled seeds treated with or without $\mathrm{GA}_{3}$ began to germinate on day 3 and day 4 (Fig. 1A and B). The total germination rate, germination index and germination potential of dehulled seeds were higher than those of hulled seeds, and both reached their maximum values after treatment with $200 \mu \mathrm{g} / \mathrm{L} \mathrm{GA}_{3}$, with the values increasing by $97.98 \%, 77.47 \%$ and $157.03 \%$, respectively, compared to the control (Fig. 1C - E).

Transcriptomic analysis of $L$. chinensis seeds soaked with $\mathrm{GA}_{3}$

We generated a total of $118.22 \mathrm{~Gb}$ of valid bases with Q30 values ranging from $95.27 \% \sim 96.72 \%$, and the mean GC content was $54.06 \%$ (Table S1). After de novo assembly by the Trinity package, we obtained a total of 203,776 transcripts and 37,208 genes with a GC content of $48.96 \%$ and $49.03 \%$, respectively. The N50 of the genes was 1,541, the total number of assembled bases was 40,055,874 (Table S2), and the maximum, minimum and median lengths of the genes were 19,928, 201 and 806, respectively. We adopted the criteria $\left|\log _{2} \mathrm{FC}\right|>1$ and false discovery rate (FDR) $\leq 0.05$ to screen differentially expressed genes (DEGs) in hulled and de-hulled seeds treated with $\mathrm{GA}_{3}$ and distilled water. A total of 4,327 DEGs of LGA vs LS were screened out, of which 2,275 genes were upregulated and 2,052 genes were downregulated (Fig. 2). Moreover, 11,919 DEGs of FGA vs FS were screened from the hulled seeds, of which 8,067 were upregulated and 3,852 were downregulated. In addition, 325 upregulated genes and 440 downregulated genes among these genes were co-expressed in both LGA vs LS and FGA vs FS.

Gene Ontology (GO) analysis was performed in this study to analyse the functions of the DEGs $(p<0.05)$. From the data shown in Table S3, a total of 6,913 and 2,378 genes were annotated in three GO functions in FGA vs FS and LGA vs LS, respectively. In the biological process category, oxidation-reduction process (GO: 0055114, 341 genes), metabolic process (GO: 0008152, 292 genes) and translation (GO: 0006412, 290 genes) were the most enriched in the comparison group FGA vs FS, while the top three enriched GO 
0055114, 121 genes) and response to cadmium ion (GO: 0046686, 64 genes). There were 362 and 219 genes enriched in the cellular component category (GO: 0005575) in FGA vs FS and LGA vs LS, respectively. Furthermore, metal ion binding (GO: 0046872, 453 genes), nucleotide binding (GO: 0000166,364 genes) and structural constituent of ribosome (GO: 0003735, 328 genes) were the top three enriched terms in FGA vs FS, and molecular function (GO: 0003674, 287 genes); hydrolase activity, hydrolyzing O-glycosyl compounds (GO: 0004553, 37 genes); and peroxidase activity (GO: 0004601, 35 genes) were the top three terms in LGA vs LS.

Kyoto Encyclopedia of Genes and Genomes (KEGG) pathway enrichment analysis revealed that DEGs in LGA vs LS seeds of $L$. chinensis were mainly related to the starch and sucrose metabolic pathway, phenylpropane biosynthesis pathway, sugar metabolic pathway, a-linolenic acid metabolic pathway, ABC transporter pathway and photosynthesis protein pathway (Fig. 3A), while the DEGs in FGA vs FS were enriched in the ribosome pathway, phenylpropane biosynthesis pathway, phagocytosis pathway, energy metabolism pathway, amino acid metabolism pathway and phosphatidylinositol signalling system pathway (Fig. 3B). The DEGs with similar regulatory trends in both LGA vs LS and FGA vs FS were also screened, and these genes were mainly enriched in protein processing in the endoplasmic reticulum, spliceosome, starch and sucrose metabolism, endocytosis and ribosome (Fig. 3C).

Validation of RNA-seq data by qRT-PCR

To further determine the accuracy of the RNA sequencing results, ten DEGs were selected randomly for qRT-PCR, and specific primers for these genes were designed by Primer 6.0 software (Table S4). The qRTPCR results were basically consistent with our transcriptome data, which proved that the data were reliable (Fig. S1).

Metabolic analysis in seeds treated with $\mathrm{GA}_{3}$

To fully understand the metabolic changes in response to $\mathrm{GA}_{3}$-mediated disruption of the seed dormancy of $L$. chinensis, a non-target metabolic analysis was performed using UPLC-qTOF-MS, and principal component analysis (PCA) of the whole samples (Fig. S2A) showed that the same treatments were gathered together, indicating good repeatability between samples, while different treatments were separated from each other, indicating that there were different effects on metabolites between treatments. Each treatment group was separated by the first component (PC1), which means that the treatment was the most important factor causing differences in metabolites rather than random errors (Fig. S2B and C). To understand the effects of the differentially abundant metabolites of $\mathrm{GA}_{3}$ on the germination of $L$. chinensis seeds, we identified 650 and 871 significantly different metabolites in FGA vs FS and LGA vs LS, respectively (Fig. 4A and B). In addition, 1221 significantly different metabolites were also screened out in LGA vs FGA to consider the influence of the hulls (Fig. 4C).

Comparative analysis between the treatments of hulled seeds of L. chinensis with $\mathrm{GA}_{3}$ and distilled water showed a significant difference in metabolites, and the significantly enriched pathways included Loading [MathJax]/jax/output/CommonHTML/fonts/TeX/fontdata.js ;tarch and sucrose metabolism, arginine and 
proline metabolism, amino sugar and nucleotide sugar metabolism, and glyoxylate and dicarboxylate metabolism (Fig. 5A). However, the main pathways in the de-hulled seeds included isoquinoline alkaloid biosynthesis; alanine, aspartate and glutamate metabolism; tyrosine metabolism; starch and sucrose metabolism; arginine and proline metabolism; and amino sugar and nucleotide sugar metabolism (Fig. 5B). Due to the differences in the main metabolic pathways associated with $\mathrm{GA}_{3}$ treatment of hulled and de-hulled seeds, the pathways associated with the hulls of $L$. chinensis seeds were also analysed (Fig. 5C and Table S5). The main differentially abundant metabolite pathways were arginine and proline metabolism, pantothenate and CoA biosynthesis, phenylpropanoid biosynthesis, alanine, aspartate and glutamate metabolism, which mainly synthesize some organic acids and amino acids, such as Larginine, pantothenate and oxoglutaric acid. It could be seen from the clustering heat map analysis (Fig. 6) of the main differentially abundant metabolites and the data in Table S6 that the abundance of the metabolites was significantly affected in hulled and de-hulled seeds of $L$. chinensis after soaking in $\mathrm{GA}_{3}$. Compared with seeds soaked in water, the content of malonic acid and citramalic acid significantly increased (2.16- and 2.18-fold in FGA; 16.68- and 34.17-fold in LGA). The levels of carbohydrates such as D-fructose, D-fructose 6-phosphate, D-glucose and D-glucose 1-phosphate were significantly increased in FGA, and they were also increased in LGA. In addition, the levels of most amino acids, such as L-tyrosine, L-histidine and L-arginine, were significantly increased in LGA, while the number of significantly enriched amino acids decreased in FGA.

Integrative analysis of DEGs and metabolites in starch and sucrose metabolism in seeds soaked with $\mathrm{GA}_{3}$

Starch and sucrose metabolism provides energy for seeds during germination, and $\mathrm{GA}_{3}$ treatment significantly influenced this pathway. Therefore, we performed an association analysis between DEGs and metabolites (a-D-glucose-1P, D-fructose, D-glucose and a-D-glucose-6P) in the starch and sucrose metabolic pathways (Fig. 7). According to the results, 5 genes encoding a-glucosidase ( $X Y L 1)$ and 6 genes encoding $\beta$-fructofuranosidase (1-SST) were upregulated in de-hulled seeds after treatment with $\mathrm{GA}_{3}$, but most of these genes were downregulated in hulled seeds. At the same time, two genes, namely, PGM2 (TRINITY_DN79669_CO_g2) and PGM (TRINITY_DN72581_CO_g6), encoding phosphoglucomutase, were upregulated in hulled seeds but exhibited very low expression in de-hulled seeds. In the process of starch hydrolysis, 2 genes and 1 gene encoding 1,4-a-glucan branching enzymes were significantly upregulated in FGA and LGA, respectively. In addition, 9 genes encoding a-amylase ( $A M Y 1.1, A M Y 1.2$ and $A M Y 1.6)$ were significantly upregulated, and among them, $A M Y 1.1$ (TRINITY_DN91755_c0_g1) had the highest expression level, which was 18.05 and 3.40 times higher in hulled and de-hulled seeds, respectively, under the $\mathrm{GA}_{3}$ treatment than in the control. Most genes encoding cellulose were downregulated under $\mathrm{GA}_{3}$ treatment in hulled and de-hulled seeds compared with the seeds treated with sterile water, while 7 of 8 genes encoding $\beta$-glucosidase (GLIP5) showed significantly enhanced expression in de-hulled seeds. In addition, the trehalose 6-phosphatase synthase gene tpsA (TRINITY_DN58656_CO_g1), SPAC2E11.16c (TRINITY_DN75474_C2_g1) and the trehalose 6-phosphatase 
phosphatase gene TPP2 (TRINITY_DN85021_CO_g1) were all significantly downregulated in hulled and de-hulled seeds, while 3 genes encoding a-trehalase (treh) were upregulated.

We also constructed a diagram of the regulatory network to clearly depict the mechanism of $\mathrm{GA}_{3}$ mediated disruption of seed dormancy through starch and sucrose metabolism using the DEGs and metabolites with similar regulatory trends in both FGA vs FS and LGA vs LS (Table S7). As shown in Fig. 8, exogenous $\mathrm{GA}_{3}$ could disrupt seed dormancy by promoting the expression of $A M Y 1.1, A M Y 1.2$, $A M Y 1.6$ and GLIP5 and inhibiting cellulose (Cel61a, egID and cel1), tpsA, SPAC2E11.16c and TPP2, thereby reducing the synthesis of maltose, cellobiose and trehalose accompanied by an increase in the glucose content and finally providing energy to $L$. chinensis seeds for disruption of dormancy.

\section{Discussion}

Effects of $\mathrm{GA}_{3}$ on seed germination of $L$. chinensis

The germination rate reflects the dynamic relationship between the seed germination rate and time. Germination potential is an indicator that reflects and explains the germination speed of seeds and can accurately reflect whether the seeds germinate in an orderly manner or not, and the germination index is an indicator of whether the germination rate is consistent. Generally, the higher the germination potential and germination index are, the better the germination regularity and germination rate of the seeds. Based on the whole germination process, $\mathrm{GA}_{3}$ treatment significantly promoted the germination rate of hulled and de-hulled $L$. chinensis seeds, and the best effect was obtained at a $\mathrm{GA}_{3}$ concentration was $200 \mu \mathrm{g} / \mathrm{L}$ (Fig. 1). Therefore, $\mathrm{GA}_{3}$ treatment can significantly promote the germination of $L$. chinensis seeds breaking dormancy; research by Cui [24] also verified these results. In addition, the hulls act as an obstacle in the process of seed germination of $L$. chinensis, and in the case of exogenous $\mathrm{GA}_{3}$ addition, the effects of hulls on $\mathrm{GA}_{3}$ also play an obstacle-like role. After removing the hulls, the germination rate of the $L$. chinensis seeds significantly improved, which is consistent with the research of Ma et al. [25].

Genetic regulation of seed germination with $\mathrm{GA}_{3}$ treatment

Seed germination is a complex biological process regulated by a large number of genes. In a suitable environment, seeds restore metabolic activity, and the enzymes and metabolites stored in the seeds are rapidly activated. After absorption of water, this series of processes involves a large amount of gene regulation and energy supply [26]. In this study, we performed a transcriptomic analysis of hulled and dehulled seeds treated with $\mathrm{GA}_{3}$ and distilled water and generated a total of 37,208 genes. After functional annotation and screening, numerous DEGs involved in $\mathrm{GA}_{3}$ treatment improved the germination of $L$. chinensis seeds, of which 6,913 and 2,378 genes were annotated in three GO functions in FGA vs FS and LGA vs LS, respectively (Table S3). Based on the $G 0$ annotation results, the oxidation-reduction process (GO: 0055114) and cellular component (GO: 0005575) terms were both significantly enriched in FGA vs FS and LGA vs LS. It has been reported that energy metabolism mediated by redox activity may be Loading [MathJax]/jax/output/CommonHTML/fonts/TeX/fontdata.js ion [27]. Meanwhile, cellular components (such 
as membrane, ribosome, nucleoplasm, etc.) are also closely related to cell division, elongation or radicle emergence during seed germination. Moreover, the KEGG pathway analysis showed that protein processing in the endoplasmic reticulum, spliceosome, starch and sucrose metabolism, endocytosis and ribosome were significantly enriched pathways in both LGA vs LS and FGA vs FS (Fig. 3), indicating that these pathways play an important role in promoting seed germination under $\mathrm{GA}_{3}$ treatment.

\section{Effects of $\mathrm{GA}_{3}$ on hulls of Leymus chinensis seeds}

Seed hulls are one of the key factors that restrict seed germination, and the mechanical properties of seed hulls may have a certain obstacle-like effect on the exchange of gas and water [28]. He et al. [29] mentioned in their study on seed dormancy of L. chinensis that seed hulls accounted for $28.4 \%$ of the causes of dormancy induction, and hulled seeds treated with $\mathrm{GA}_{3}$ also exhibited a significantly reduced percentage of seed dormancy. Therefore, in this study, the main metabolic pathways involved in the treatment of hulls after $\mathrm{GA}_{3}$ treatment and the main differentially abundant metabolites involved were explored. Comparison of the $\mathrm{GA}_{3}$-treated hulled and de-hulled seeds (Fig. 5 and Table S5) showed the main metabolites in hulls were L-arginine and feruloylputrescine in the pathway of arginine and proline metabolism and 2-dehydroepianate and pantothenic acid in the pathway of pantothenate and CoA biosynthesis, which are key metabolites of synthetic organic acids. Similar findings were also reported by $\mathrm{Yu}$ [30], who studied the substances inhibiting seed germination and seedling growth in various parts of Taxus chinensis var. mairei seeds and found that organic acids, esters and alcohols are distributed in seed hulls. Similarly, germination-inhibiting organic acids were also detected in Torreya grandis seed hulls [31]. The organic acids in the hulls of seeds regulate the seed dormancy mechanism through the metabolism of arginine and proline and the synthesis of pantothenic acid and coenzyme A. However, arginine is an important amino acid synthesis substrate in the tricarboxylic acid (TCA) cycle. In this study, the TCA cycle also appeared in the hulls treated with $\mathrm{GA}_{3}$, which may be due to $\mathrm{GA}_{3}$ treatment providing energy for reducing the synthesis of germination inhibitors in the hulls.

Genes and metabolites in starch and sucrose metabolic pathways of Leymus chinensis seeds treated with $\mathrm{GA}_{3}$

Seeds need much energy during the process of dormancy disruption and germination, including for the synthesis of DNA and cell walls. There has been much speculation regarding the sources of energy and the associated metabolic process. In the process of seed germination, GA can effectively regulate the activity of seed metabolism and the expression of genes and metabolites related to seed germination, thus promoting the shift from seed dormancy to germination. Based on our results, starch and sucrose metabolism is a significantly enriched pathway in seeds treated with $\mathrm{GA}_{3}$ and could provide energy for seeds during the process of dormancy disruption. The main metabolites that exhibited increased levels in the hulled and de-hulled seeds treated with $\mathrm{GA}_{3}$ were $\mathrm{a}$-D-glucose-1P, D-fructose, D-glucose and $\mathrm{a}-\mathrm{D}$ glucose-6P in the starch and sucrose metabolic pathways, and these metabolites may eventually 
participate in glycolysis, the TCA cycle or the pentose phosphate pathway to provide energy for seed germination.

According to our results (Fig. 7), GA 3 promoted the upregulation of the genes a-glucosidase ( $X Y L 7$ ) and $\beta$ fructofuranosidase (1-SST) in de-hulled seeds of L. chinensis, and sucrose was hydrolysed to D-fructose via the action of $X Y L 1$ and $1-S S T$, but these two genes were downregulated in hulled seeds. At the same time, a downstream product of sucrose, a-D-glucose-1P, was hydrolysed to a-D-glucose-6P under the action of two upregulated genes, namely, PGM2 (TRINITY_DN79669_CO_g2) and PGM

(TRINITY_DN72581_CO_g6), in hulled seeds, but the expression of these genes was very low expression in de-hulled seeds; these genes encode phosphoglucomutase and are further involved in the glycolysis pathway. It has been reported that sucrose may be an intermediate of plant metabolism, and the decrease in sucrose decomposition results in low levels of glucose and fructose and inhibits seed germination [32].

In addition to sucrose, the decomposition of starch, cellulose and trehalose also provides energy for seed germination. Starch is a polysaccharide stored in plant seeds that can be converted into reducing sugars (such as maltose and glucose) by amylase and plays an important role in seed germination [33]. The seed embryo needs nutrition during the development of seed morphology, and these nutrients are obtained not only by transformation of storage substances in the endosperm but also via the metabolism of nutrients in the embryo, which provide the energy needed for embryonic development. Li et al. [34] reported that the starch content in the endosperm is dynamic, and starch is hydrolysed to sugar by amylase, providing energy for seed germination. The a-amylase not only converts starch to glucose and other reducing sugars by acting on the a-1,4-glycosidic bond but also reduces the viscosity of starch [35, 36]. When the seed germinates, the expression level of the a-amylase gene increases, and the starch stored in the seed is hydrolysed. The a-amylase gene is a downstream target gene for GA-mediated regulation of seed germination and encodes an enzyme that hydrolyses starch in the endosperm. GA can induce the expression of the amylase gene and then promote starch hydrolysis via the GA response element [37]. Studies have shown that the transcription level of the a-amylase gene can be regulated, and GAMyb-type transcription factors play a key role in this process. GA regulates the expression of GAMyb in a DELLA-dependent manner. GAMyb binds to the GA response element on the promoter of the a-amylase gene and activates its expression [38]. In this study, 9 genes encoding a-amylase (AMY1.1, AMY1.2 and AMY1.6) were significantly upregulated, and among them, AMY1.1 (TRINITY_DN91755_CO_g1) was upregulated 18.05- and 3.40-fold in hulled and de-hulled seeds, respectively. Under the action of aamylase and the 1,4-a-glucan branching enzyme, amylose and starch stored in seeds can be hydrolysed to maltose and subsequently broken down to glucose by a-glucosidase. Cellulose is a key component of the plant cell wall. Cellulase cleaves cellulose to form cellobiose or cellodextrin, and then, $\beta$-glucosidase finally cleaves these two hydrolysates to glucose [39]. In this study, most genes encoding cellulase were downregulated under treatment with $\mathrm{GA}_{3}$ in hulled and de-hulled seeds compared with the group treated with sterile water, which inhibited the decomposition of cellulose, while 7 of 8 genes encoding $\beta$ glucosidase (GLIP5) showed significantly enhanced expression in de-hulled seeds, breaking down cellodextrin and cellobiose to qlucose. This is likelv because elongation of the radicle cannot occur Loading [MathJax]/jax/output/CommonHTML/fonts/TeX/fontdata.js

Page 9/24 
without cell wall remodelling, and on the other hand, decomposition of cellodextrin and cellobiose could also produce more D-glucose.

Trehalose is a non-reducing disaccharide that can be used as an energy source for glycolysis and is associated with various types of stress tolerance. The trehalose 6-phosphatase synthase gene and atrehalase gene have opposite functions, regulating trehalose synthesis and degradation, respectively [40]. In seeds of $L$. chinensis after $\mathrm{GA}_{3}$ treatment, the trehalose 6-phosphatase synthase gene tpsA (TRINITY_DN58656_CO_g1), SPAC2E11.16c (TRINITY_DN75474_c2_g1) and the trehalose 6-phosphatase phosphatase gene TPP2 (TRINITY_DN85021_CO_g1) were all significantly downregulated, which inhibited the synthesis of trehalose. Meanwhile, 3 genes encoding a-trehalase (treh) were upregulated, hydrolysing trehalose to glucose. This is consistent with observation that the trehalose content decreased during spore germination in some fungal species [41]. Trehalose may act as a buffer regulating the intracellular level of glucose and may begin to degrade when the intracellular glucose concentration is insufficient [42].

\section{Conclusions}

In this study, through transcriptomics and metabolomics, we analysed the molecular mechanism by which $\mathrm{GA}_{3}$ disrupts seed dormancy in $L$. chinensis. The results revealed that exogenous $\mathrm{GA}_{3}$ can significantly promote seed germination by regulating some important genes and their related metabolites. Starch and sucrose metabolism is one of the most highly enriched pathways that may play a key role in energy supply for the transition of $L$. chinensis seeds from the dormant state to germination by suppressing the expression of Cel61a, egID, cel1, tpSA, SPAC2E11.16c and TPP2, along with enhancing the expression of $A M Y 1.1, A M Y 1.2, A M Y 1.6$ and GLIP5, and finally inhibiting the synthesis of cellobiose, cellodextrin, and trehalose while promoting the hydrolysis of sucrose, starch, cellobiose, cellodextrin, and trehalose to glucose. These findings provide insights for understanding the mechanisms by which $\mathrm{GA}_{3}$ disrupts dormancy and provide valuable information for further breeding of $L$. chinensis varieties with high germination rates.

\section{Materials And Methods}

Plant materials and seed treatments

Seeds of L. chinensis were collected from the Songnen Grassland with the permission of the Grassland Station of Daqing in Heilongjiang province of China. Heilongjiang Frigid Zone Plant Gene Resource Research Center undertook the formal identification of the samples and provided details of specimen deposited. Mature and plump seeds were divided into two types: hulled seeds and de-hulled seeds (hulls were peeled off by hand). All seeds were disinfected for $5 \mathrm{~min}$ in $5 \%$ sodium hypochlorite, followed by rinsing with $75 \%$ alcohol twice. After rinsing three times using sterile water, the seeds were dried at room temperature. 
The hulled and de-hulled seeds (100 mg per sample) were soaked in $50 \mathrm{ml}$ of $\mathrm{GA}_{3}$ solution at different concentrations $(100,200$ and $300 \mu \mathrm{g} / \mathrm{L})$ for $24 \mathrm{~h}$ at $25^{\circ} \mathrm{C}$, and the control groups were soaked in sterile water. The concentration gradient of $\mathrm{GA}_{3}$ was based on a previous study [43]. All treatments were performed three times.

Seed germination assays

A total of $100 \mathrm{mg}$ of sterilized seeds (approximately 50 seeds) was evenly placed in each culture dish. Culture dishes were placed in a room at $28^{\circ} \mathrm{C}$ (light) or $19^{\circ} \mathrm{C}$ (dark) for $12 \mathrm{~h}$, and the seedbed was kept moist by watering regularly during germination. The number of germinated seeds was counted daily for 21 days to calculate the germination rate for the first 7 days and the total germination rate. Germination potential was the germination number on the 6th day/the total number of seeds, and the germination index was calculated by the following equation (where $\mathrm{Gt}$ is the germination number at different times ( 7 days), and $D t$ is the number of days of germination):

Germination index $=\sum \mathrm{Gt} / \mathrm{Dt}$

Samples for transcriptomic and metabolomic analyses

The concentration of $\mathrm{GA}_{3}(200 \mu \mathrm{g} / \mathrm{L})$ used was determined from the former germination test. Mature and plump seeds of $L$. chinensis with and without hulls were first disinfected and then soaked in $200 \mu \mathrm{g} / \mathrm{L}$ $\mathrm{GA}_{3}$ solution for $24 \mathrm{~h}$, defined as FGA and LGA, respectively; another set of seeds was soaked in sterile water for $24 \mathrm{~h}$, defined as FS and LS, respectively. After the treatments, seeds were placed in culture dishes to germinate at room temperature for $72 \mathrm{~h}$. All the treatments were performed three times. Transcriptomic and metabolomic analyses were carried out by LC-Bio Technologies (Hangzhou) Co., Ltd.

RNA extraction, quality control and RNA-seq

The OminiPlant RNA Kit (CWBIO, China) was used to extract total RNA from each sample following the manufacturer's procedure. Total RNA was checked for quantity and purity using a Bioanalyzer 2100 and an RNA 1000 Nano LabChip Kit (Agilent, CA, USA) with RIN number > 7.0. Two rounds of purification were used to purify poly(A) RNA from $5 \mu \mathrm{g}$ of total RNA using poly-T oligo-attached magnetic beads. Then, the mRNA was fragmented into small fragments using divalent cations at a high temperature. The cDNA library was created via reverse transcription using the mRNA-Seq Sample Preparation Kit (Illumina, San Diego, USA), and the paired-end libraries were constructed with an average insert fragment size of $300 \mathrm{bp}$ ( $\pm 50 \mathrm{bp}$ ). Finally, paired-end transcriptome sequencing of $L$. chinensis seeds was performed on an Illumina HiSeq4000 platform (LC Sciences, USA) using recommended protocols.

De novo assembly and functional annotation

The reads in the sequencing data that contained low-quality bases, adaptor contamination, and undetermined bases were first removed using cutadapt [44] and in-house Perl scripts. Then, FastQC Loading [MathJax]/jax/output/CommonHTML/fonts/TeX/fontdata.js tqc/) was used to check the sequence quality, 
including the Q20, Q30 and GC content of the clean data. All further analyses in this study were based on high-quality clean data. Trinity (version 2.4.0) [45] was employed for de novo assembly of our transcriptomic data and for grouping transcripts into clusters on the basis of shared sequence content. Each transcript cluster was very loosely defined as a 'gene', and the longest transcript in the cluster was taken as the 'gene' sequence.

DIAMOND [46] was used with an E-value threshold $<0.00001$ to annotate these assembled unigenes by aligning against the nonredundant protein (Nr) (http://www.ncbi.nlm.nih.gov/), GO (http://www.geneontology.org), SwissProt (http://www.expasy.ch/sprot/), KEGG (http://www.genome.jp/kegg/) and eggnog (http://eggnogdb.embl.de/) databases. Transcript per million (TPM) [47] values were calculated using Salmon [48] to reflect the unigene expression levels. Differentially expressed unigenes were screened using the R package edgeR [49] with the criteria of FDR $\leq 0.05, \log 2$ (fold change) $>1$ or log2 (fold change) $<-1$. Then, GO and KEGG pathway enrichment analyses of differentially expressed unigenes were carried out again by using in-house Perl scripts.

Validation of transcriptomic data for real-time quantitative reverse transcription PCR (qRT-PCR)

To verify the accuracy of our transcriptomic data, ten DEGs involved in L. chinensis seed dormancy disruption were randomly selected for qRT-PCR, and the specific primers for these genes were designed by Primer 6.0 (Table S4). The synthesis of first-strand cDNA was performed using the TUREscript 1st Strand cDNA Synthesis Kit (Aidlab, China). The reaction contained cDNA (1000 ng), $5 \times$ RT Reaction Mix (4 $\mu \mathrm{L})$, random primer/oligodT $(0.8 \mu \mathrm{L})$, and TUREscript $\mathrm{H}^{-}$RTase/RI Mix $(0.8 \mu \mathrm{L})$, and RNase Free $\mathrm{dH}_{2} \mathrm{O}$ was added to obtain a final volume of $20 \mu \mathrm{L}$. The qRT-PCR contained $5 \mu \mathrm{L}$ of $2 \times S Y B R \circledast$ Green Supermix (DF, China), $0.5 \mu \mathrm{L}$ of forward primer, $0.5 \mu \mathrm{L}$ of reverse primer, $1 \mu \mathrm{L}$ of cDNA, and $3 \mu \mathrm{L}$ of $\mathrm{ddH}_{2} \mathrm{O}$. qRT-PCR was conducted on an Analytik Jena qTOWER2.2 instrument (Germany) with the following program: $95^{\circ} \mathrm{C}$ for $3 \mathrm{~min}$, followed by $39 \mathrm{cycles}$ of $95^{\circ} \mathrm{C}$ for $10 \mathrm{~s}$ and $58^{\circ} \mathrm{C}$ for $30 \mathrm{~s}$. All the samples were examined in triplicate with four technical replicates. The relative expression of genes was calculated by the $2^{-\Delta \Delta C t}$ method using actin as the reference gene [50].

Metabolite extraction and metabolic spectrum analysis

The collected samples were first thawed on ice, and then, $120 \mu \mathrm{L}$ of precooled $50 \%$ methanol buffer was added into $20 \mu \mathrm{L}$ of sample followed by 1 min of vortexing and 10 min of incubation at room temperature. The extraction mixture solution was stored overnight at $-20^{\circ} \mathrm{C}$ in a freezer and then centrifuged at 4,000 r/min for $20 \mathrm{~min}$. The supernatants were transferred into 96 -well plates and stored at $-80^{\circ} \mathrm{C}$ for subsequent LC-MS analysis. Furthermore, $10 \mu \mathrm{L}$ of each extraction mixture was combined into a pooled quality control (QC) sample.

In this study, 43 samples of L. chinensis seeds (including QC samples) were detected by a TripleTOF 5600 system in positive and negative ion modes, and the mass spectrum data were interpreted by combining with biological information analysis. The biological information analysis mainly used XCMS Loading [MathJax]/jax/output/CommonHTML/fonts/TeX/fontdata.js letex software was used to screen quantitative 
and differentially abundant substances. Metabolites were annotated in HMDB, KEGG and other databases.

The first-level mass spectrometry information was used for identification, and the second-level mass spectrometry information was used for matching with the in-house standard database. In this paper, the original mass spectrometry data were transformed to the readable $\mathrm{mzXML}$ data format by using the msconvert tool of Proteowizard software.

Metabolic pathway construction

Three-dimensional data obtained in this study, including sample name, peak number and normalized data, were input into SIMCA software (V14, Umetrics AB, Umea, Sweden), and PCA was performed for all samples. Student's T-test $(p<0.05)$ and VIP (variable importance in the projection) values $>1$ were used to search for differentially abundant metabolites. Metabolite pathways were constructed by searching noncommercial databases, such as KEGG (http://www.genome.jp/kegg/) and NIST (http://www.nist.gov/index.html).

\section{Statistical analysis}

Statistical analysis was performed by one-way ANOVA, and multiple comparisons were conducted using Duncan's multiple comparisons test in SAS 9.0 (SAS Institute, Cary, USA). Data are shown as the means \pm $\operatorname{SDs}(n=3)$.

\section{Abbreviations}

FGA

hulled seeds soaked in $200 \mu \mathrm{g} / \mathrm{L} \mathrm{GA}_{3}$ solution for $24 \mathrm{~h}$; LGA:de-hulled seeds soaked in $200 \mu \mathrm{g} / \mathrm{L} \mathrm{GA}_{3}$ solution for $24 \mathrm{~h}$; FS:hulled seeds soaked in sterile water for $24 \mathrm{~h}$; LS:de-hulled seeds soaked in sterile water for $24 \mathrm{~h} ; \mathrm{GA}_{3}$ :gibberellic acid; DEGs:differentially expressed genes; Nr:nonredundant protein database; FDR:false discovery rate; GO:Gene Ontology; KEGG:Kyoto Encyclopedia of Genes and Genomes; TPM:transcripts per million; PCA:principal component analysis.

\section{Declarations}

\section{Acknowledgements}

We are grateful to American Journal Experts (AJE) for providing language editing services for this manuscript.

\section{Funding}

This work was supported by the National Key Research and Development Project of China Loading [MathJax]/jax/output/CommonHTML/fonts/TeX/fontdata.js h of the study and collection, analysis, and 
interpretation of data and in writing the manuscript.

\section{Availability of data and materials}

The RNA-seq datasets generated during the current study have been submitted to the NCBI Sequence Read Archive under the accession number PRJNA642340

(https://www.ncbi.nlm.nih.gov/sra/PRJNA642340), and other data supporting the results are included in this published article and its supplementary information files.

\section{Author contributions}

GFH and PZ designed the research. BL, FDW, RL, QNW, JL, WL and BW conducted the experiments and analysed the data. BL, PZ, FDW and RL wrote the manuscript. All authors have read and approved the manuscript.

\section{Ethics approval and consent to participate}

Not applicable.

\section{Consent for publication}

Not applicable.

\section{Competing interests}

The authors declare that they have no conflicts of interest.

\section{References}

1. Finch-Savage WE, Leubner-Metzger G. Seed dormancy and the control of germination. New Phytol. 2006;171(3):501-23.

2. Nelson SK, Ariizumi T, Steber CM. Biology in the Dry Seed: Transcriptome Changes Associated with Dry Seed Dormancy and Dormancy Loss in the Arabidopsis GA-Insensitive sleepy1-2 Mutant. Frontiers in plant science. 2017;8:2158-.

3. Tognacca RS, Servi L, Hernando CE, Saura-Sanchez M, Yanovsky MJ, Petrillo E, et al. Alternative Splicing Regulation During Light-Induced Germination of Arabidopsis thaliana Seeds. Frontiers in plant science. 2019;10:1076-.

4. Shu K, Liu XD, Xie Q, He ZH. Two Faces of One Seed: Hormonal Regulation of Dormancy and Germination. Molecular plant. 2016;9(1):34-45.

5. Lee HG, Lee K, Seo PJ. The Arabidopsis MYB96 transcription factor plays a role in seed dormancy. Plant molecular biology. 2015;87:371-81.

6. Bewley JD, Black M. Seeds. Physiology of development and germination. New York: Plenum Press; 
7. Dave A, Vaistij FE, Gilday AD, Penfield SD, Graham IA. Regulation of Arabidopsis thaliana seed dormancy and germination by 12-oxo-phytodienoic acid. J Exp Bot. 2016;67(8):2277-84.

8. Wang W, Su X, Tian Z, Liu Y, Zhou Y, He M. Transcriptome profiling provides insights into dormancy release during cold storage of Lilium pumilum. BMC Genomics. 2018;19(1):196.

9. Shu K, Zhang H, Wang S, Chen M, Wu Y, Tang S, et al. ABI4 regulates primary seed dormancy by regulating the biogenesis of abscisic acid and gibberellins in arabidopsis. PLoS Genet. 2013;9(6):e1003577-e.

10. Yang L, Jiang Z, Liu S, Lin R. Interplay between REVEILLE1 and RGA-LIKE2 regulates seed dormancy and germination in Arabidopsis. New Phytol. 2020;225(4):1593-605.

11. Mitchum MG, Yamaguchi S, Hanada A, Kuwahara A, Yoshioka Y, Kato T, et al. Distinct and overlapping roles of two gibberellin 3-oxidases in Arabidopsis development. Plant $\mathrm{J}$. 2006;45(5):804-18.

12. Hirano K, Ueguchi-Tanaka M, Matsuoka M. GID1-mediated gibberellin signaling in plants. Trends in plant science. 2008;13(4):192-9.

13. Khalil-Ur-Rehman M, Sun L, Li CX, Faheem M, Wang W, Tao JM. Comparative RNA-seq based transcriptomic analysis of bud dormancy in grape. BMC plant biology. 2017;17(1):18.

14. Zhuang W, Gao Z, Wen L, Huo X, Cai B, Zhang Z. Metabolic changes upon flower bud break in Japanese apricot are enhanced by exogenous GA4. Horticulture research. 2015;2(1):15046.

15. He X, Hu X, Wang Y. Study on Seed Dormancy Mechanism and Breaking Technique of Leymus chinensis. Acta Botanica Boreali-Occidentalia Sinica. 2010;30(1):120-5.

16. Yi J. Studies on dormancy physiology and germination rate of Leymus chinensis seeds. Grassland of China. 1994(6):1-6.

17. Ma HY, Liang ZW, Wang ZC, Chen Y, Huang LH, Yang F. Lemmas and endosperms significantly inhibited germination of Leymus chinensis (Trin.) Tzvel. (Poaceae). J Arid Environ. 2008;72(4):5738.

18. Liu G. Leymus chinensis germplasm resources research. BeiJing: Science Press; 2011.

19. Lin J, Sheng H, Shuai S, Ying W, Chunsheng M, Zhuolin L, et al. Ecological Responses of Different Maturity Leymus chinensis Seeds to Soil Burial Depths in Songnen Grassland. Acta Agrestia Sinica. 2014;22(1):52-6.

20. Liu S, Liu P, Yang W, Qi D, Li X, Liu G. The identification and expression analysis of genes related to seed germination in Leymus chinensis. Acta Prataculturae Sinica. 2018;27(11):58-66.

21. Liu S. The Function and Associated Mechanism of Gibberellin on Bud Dormancy Release in Blueberry [Dissertation]. Dalian: Dalian University of Technology 2017.

22. Chen SY, Kuo SR, Chien CT. Roles of gibberellins and abscisic acid in dormancy and germination of red bayberry (Myrica rubra) seeds. Tree physiology. 2008;28(9):1431-9.

23. Guo J, Peng L, Xu Q, Wang J. Studies on methods for breaking dormancy and dormancy feature of $\Delta$ ronitum flavum ceed Chin Arrir Sri Rull $2011 \cdot 27(10): 139-43$.

Loading [MathJax]/jax/output/CommonHTML/fonts/TeX/fontdata.js

Page 15/24 
24. Cui S. Effects of exogenous gibberellic acid $\left(\mathrm{GA}_{3}\right)$ on growth and biomass of Leymus chinensis [ Dissertation]. Chang Chun: Northeast Normal University; 2004.

25. Ma H, Liang Z, editors. Mechanical and physical stress of the outer mites significantly inhibited the germination of Leymus chinensis seeds. National Symposium on Plant Physiology and Molecular Biology; 2005. Zhan Jiang: Chinese Society of Plant Biology; 2005.

26. Wolny E, Betekhtin A, Rojek M, Braszewska-Zalewska A, Lusinska J, Hasterok R. Germination and the Early Stages of Seedling Development in Brachypodium distachyon. Int J Mol Sci. 2018;19(10):14.

27. Nietzel T, Mostertz J, Ruberti C, Nee G, Fuchs P, Wagner S, et al. Redox-mediated kick-start of mitochondrial energy metabolism drives resource-efficient seed germination. Proc Natl Acad Sci U S A. 2020;117(1):741-51.

28. Debeaujon I, L-K KM, Koornneef M. Influence of the Testa on Seed Dormancy, Germination, and Longevity in Arabidopsis. Plant Physiol. 2001;122(2):403-14.

29. He XQ, Wang YR, Hu XW, Baskin CC, Baskin JM, Lv YY. Seed dormancy and dormancy-breaking methods in Leymus chinensis (Trin.) Tzvel. (Poaceae). Grass and Forage Science. 2016;71(4):641-8.

30. Yu H. The seed dormancy mechanisms and germinating conditions of Taxus chinensis var.mairei [ Dissertation]. Beijing: Beijing Forestry University; 2009.

31. Sun N, Chen L, Liu X, Lu X, Xing L. The Effects of Germination Inhibitors Contained in Torreya grandis Seeds on Seed Germination. Journal of Huangshan University. 2018;18(3):44-7.

32. Wakabayashi T, Joseph B, Yasumoto S, Akashi T, Aoki T, Harada K, et al. Planteose as a storage carbohydrate required for early stage of germination of Orobanche minor and its metabolism as a possible target for selective control. J Exp Bot. 2015;66(11):3085-97.

33. Lunn, Major BJ, Kettlewell PS, Scott RK. Mechanisms Leading to Excess Alpha -Amylase Activity in Wheat (Triticum aestivum, L) Grain in the U.K. J Cereal Sci. 2001;33(3):313-29.

34. Li Q, Yu H, Zhou F, Guo H, Xia X, Li F. Physiological and Biochemical Characteristics of Taxus chinensis var. mairei Seeds in Dormancy Releasing Process. Guizhou Agricultural Sciences. 2012;40(6):26-9.

35. Sun H, Zhang P, Shen H. Seed Dormancy Cause of Oplopanax elatus Revealed by in vitro Culture of Excised Embryos. Seed. 2006;25(9):17-23.

36. Liu J, Zhang P, Shen H, Zhang Y, Fan S. Factors Influencing Seed Germination of Oplopanax elatus Nakai. Chinese Bulletin of Botany. 2005;22(2):183-9.

37. Jones HD, Smith SJ, Desikan R, Plakidou-Dymock S, Lovegrove A, Hooley R. Heterotrimeric G proteins are implicated in gibberellin induction of a-amylase gene expression in wild oat aleurone. Plant Cell. 1998;10(2):245-54.

38. Gubler F, Jacobsen JV. Gibberellin-responsive elements in the promoter of a barley high-pl alphaamylase gene. Plant Cell. 1992;4(11):1435-41.

39. Kubicek CP, Seidl V, Seiboth B. Plant cell wall and chitin degradation. In: Borkovish KA, Ebbole DJ, editors. Cellulose and Molecular Bioloav of Filamentous Fungi. Washington, DC, USA: ASM Press; Loading [MathJax]/jax/output/CommonHTML/fonts/TeX/fontdata.js 
2010.

40. Li ZG, Luo LJ, Zhu LP. Involvement of trehalose in hydrogen sulfide donor sodium hydrosulfideinduced the acquisition of heat tolerance in maize (Zea mays L.) seedlings. Botanical studies. 2014;55(1):20.

41. Thevelein JM, den Hollander JA, Shulman RG. Changes in the activity and properties of trehalase during early germination of yeast ascospores: correlation with trehalose breakdown as studied by in vivo 13C NMR. Proc Natl Acad Sci U S A. 1982;79(11):3503-7.

42. Pfeffer PE, Douds DD Jr, Becard G, Shachar-Hill Y. Carbon uptake and the metabolism and transport of lipids in an arbuscular mycorrhiza. Plant Physiol. 1999;120(2):587-98.

43. Liu M, Li J, Niu J, Wang R, Song J, Lv J, et al. Comparative Study on Gibberellin and Chemical Reagent on Effect of Germination Rate and Vigor of Leymus chinensis. Southwest China Journal of Agricultural Sciences. 2014;27(6):2687-91.

44. Martin M. Cutadapt removes adapter sequences from high-throughput sequencing reads. EMBnetjournal. 2011;17(1):10-2.

45. Grabherr MG, Haas BJ, Yassour M, Levin JZ, Thompson DA, Amit I, et al. Full-length transcriptome assembly from RNA-Seq data without a reference genome. Nat Biotechnol. 2011;29(7):644-52.

46. Buchfink B, Xie C, Huson DH. Fast and sensitive protein alignment using DIAMOND. Nat Methods. 2015;12(1):59-60.

47. Mortazavi A, Williams BA, McCue K, Schaeffer L, Wold B. Mapping and quantifying mammalian transcriptomes by RNA-SEq. Nat Methods. 2008;5(7):621-8.

48. Patro R, Duggal G, Love MI, Irizarry RA, Kingsford C. Salmon provides fast and bias-aware quantification of transcript expression. Nat Methods. 2017;14(4):417-9.

49. Robinson MD, McCarthy DJ, Smyth GK. edgeR: a Bioconductor package for differential expression analysis of digital gene expression data. Bioinformatics. 2010;26(1):139-40.

50. Livak KJ, Schmittgen TD. Analysis of Relative Gene Expression Data Using Real-Time Quantitative PCR and the $2-\Delta \Delta$ CT Method. Methods. 2001;25(4):402-8.

\section{Figures}



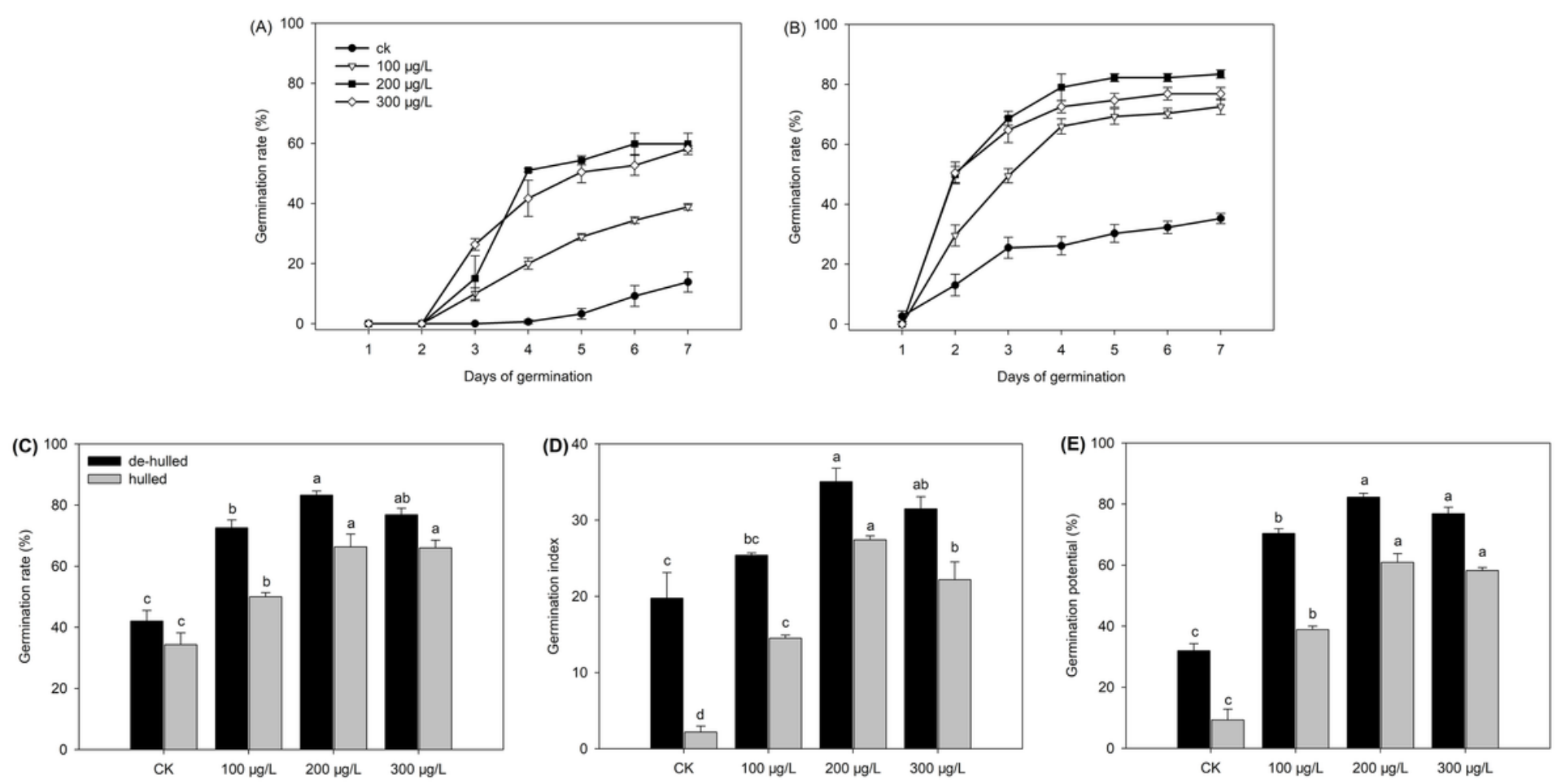

\section{Figure 1}

Effects of GA3 on the germination characteristics of L. chinensis seeds. A: Germination rate of hulled seeds in the first 7 days. B: Germination rate of de-hulled seeds in the first 7 days. C: Total germination rate at 21 days. D: Germination index. E: Germination potential.

FGA_VS_FS

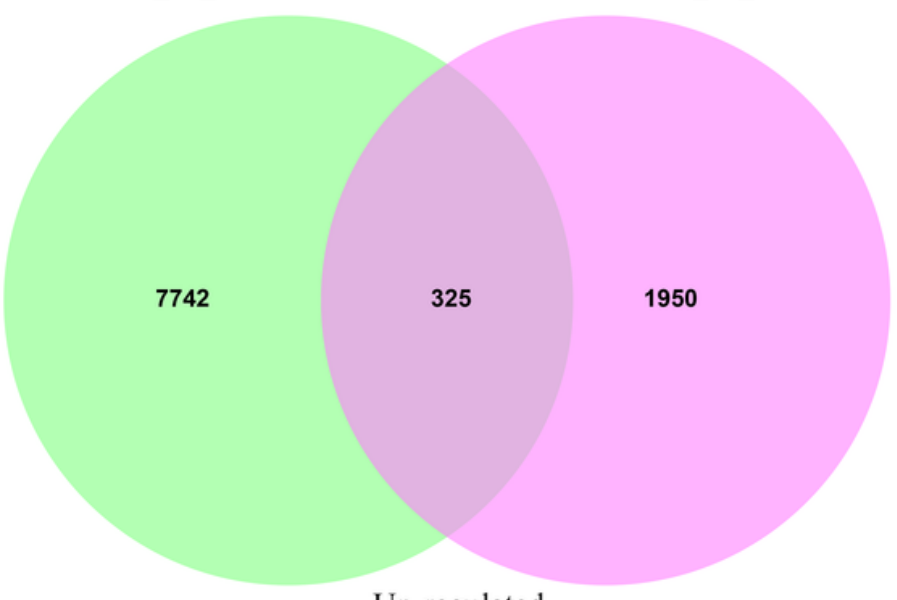

Up-regulated
FGA VS FS

LGA_VS_LS

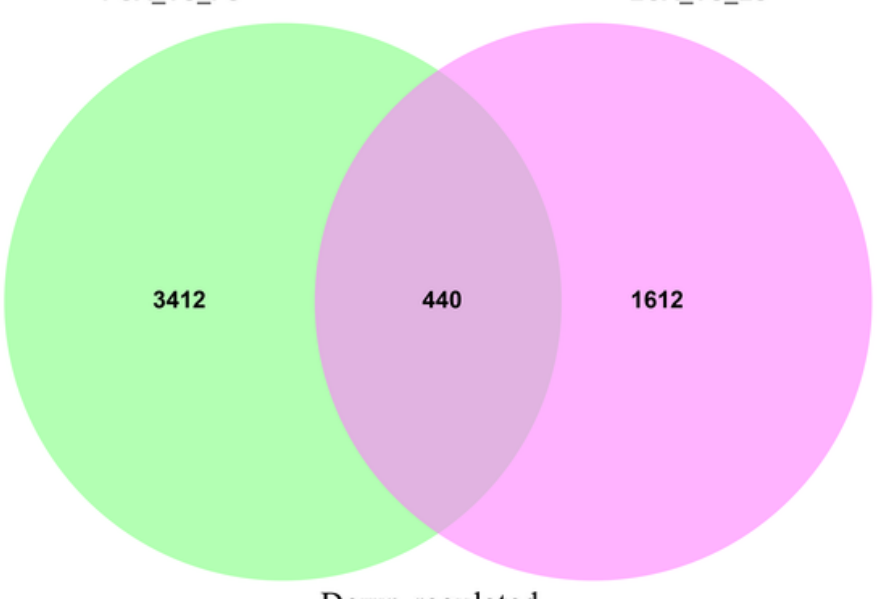

Down-regulated

Figure 2

Up- and downregulated genes in L. chinensis seeds under different treatments. LGA: de-hulled seeds soaked in $200 \mu \mathrm{g} / \mathrm{L} \mathrm{GA3}$ solution for 24 h; LS: de-hulled seeds soaked in sterile water for 24 h; FGA: hulled Loading [MathJax]/jax/output/CommonHTML/fonts/TeX/fontdata.js d seeds soaked in sterile water for $24 \mathrm{~h}$. 
(A)

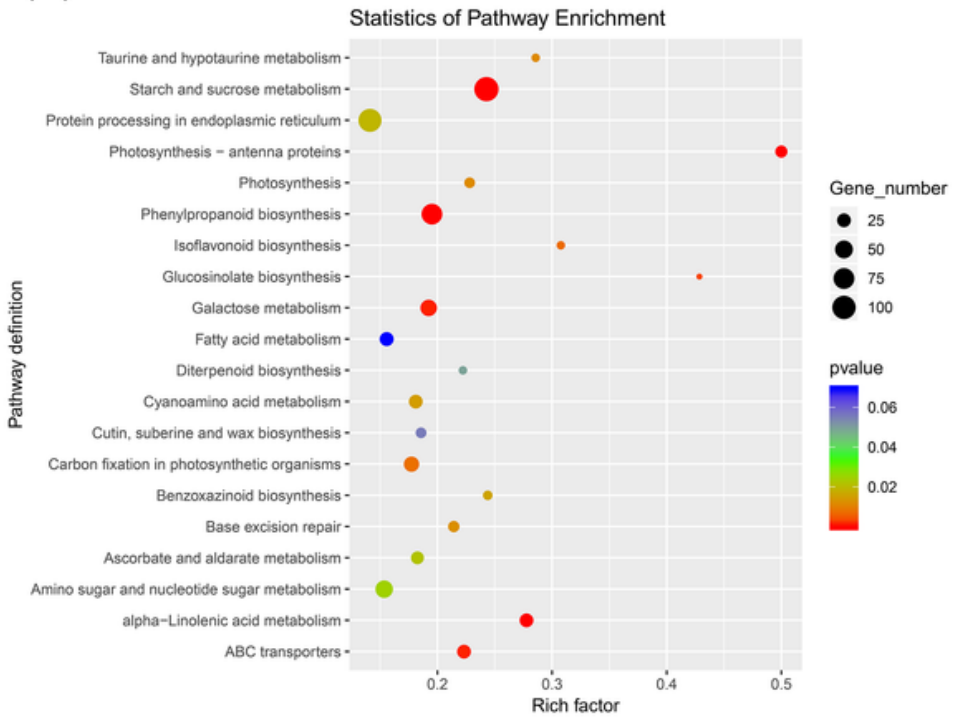

(B)

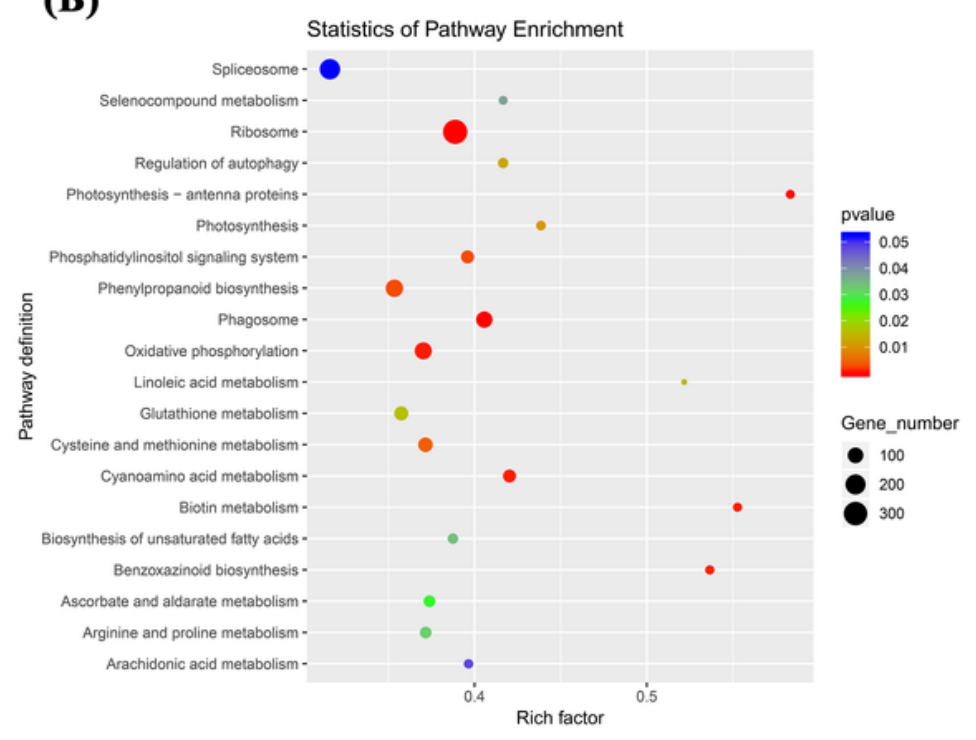

(C)

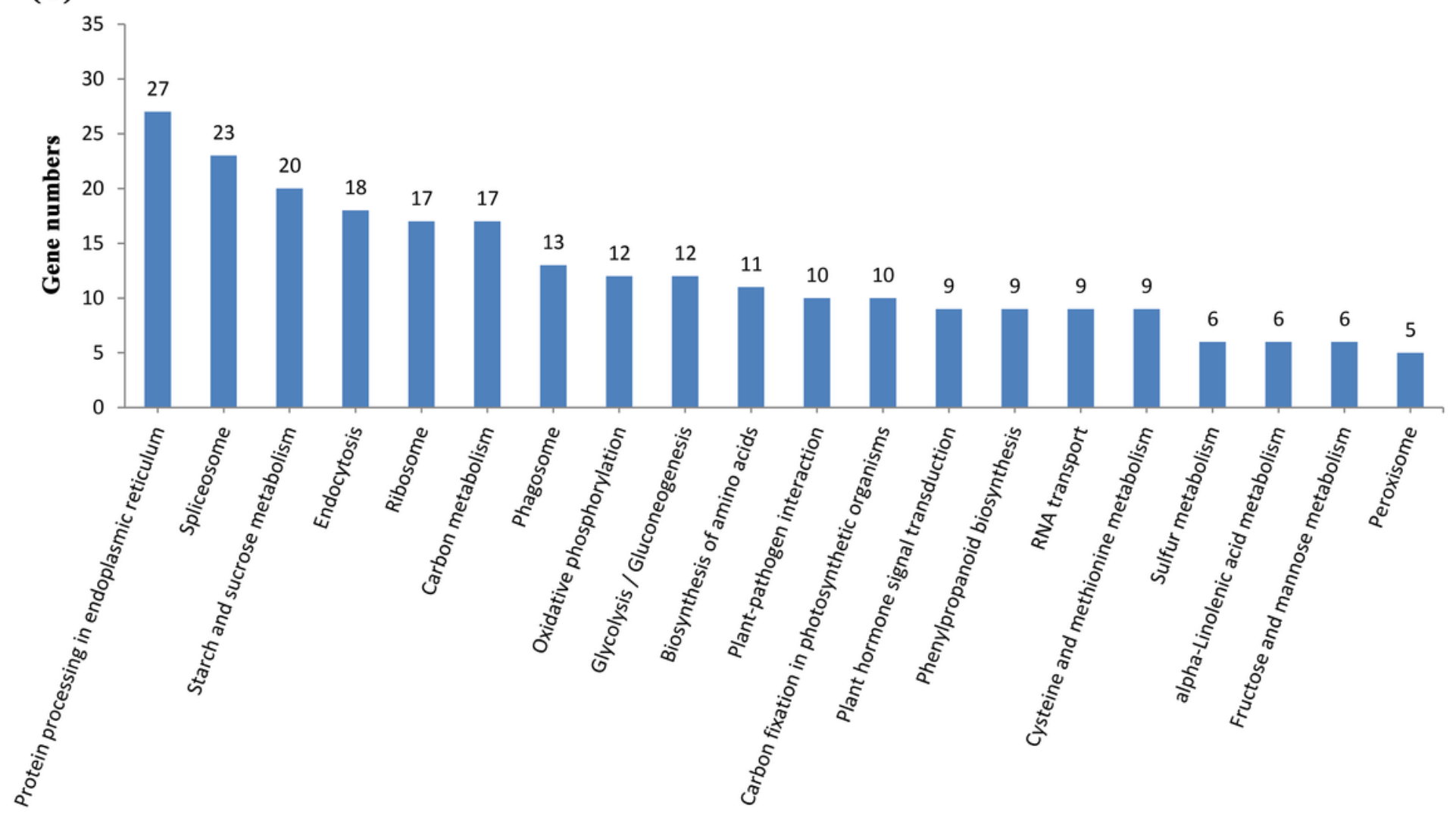

KEGG pathway

Figure 3

KEGG pathway enrichment analysis of DEGs in L. chinensis seeds under different treatments. A: LGA vs LS; B: FGA vs FS; C: DEGs with similar regulatory trends significantly enriched in both LGA vs LS and FGA vs FS. The size of the circles represents the number of genes enriched in the pathway, and the colour of the circle represents the $P$ value. 


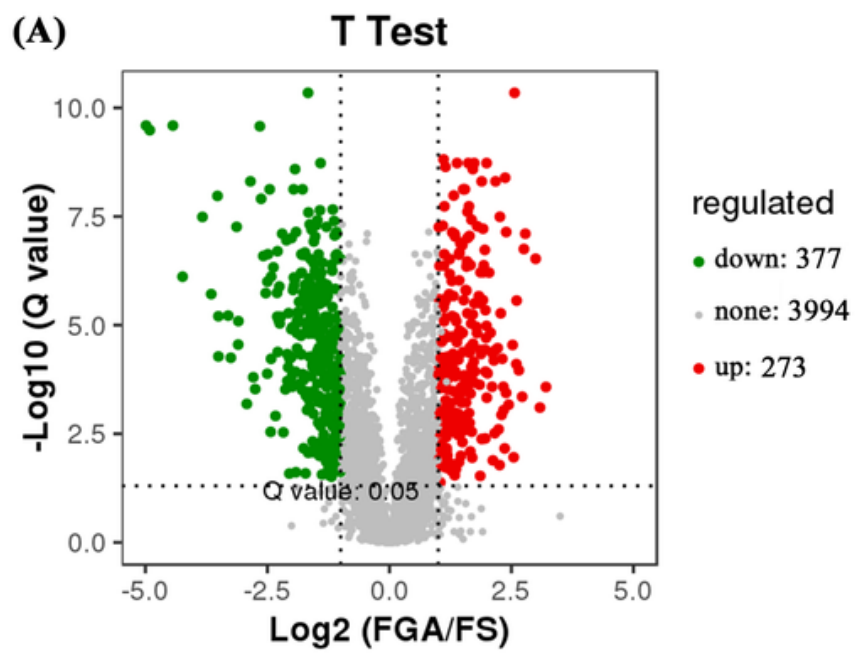

(B)

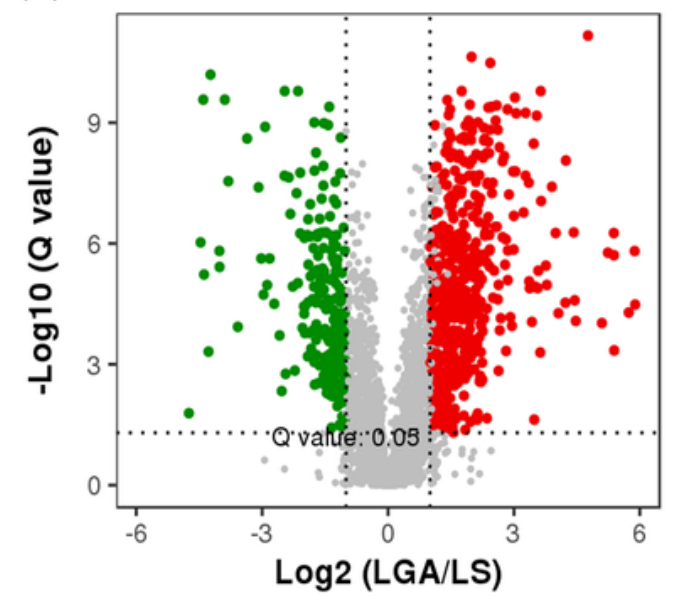

regulated

- down: 269

none: 3773

- up: 602

(C)

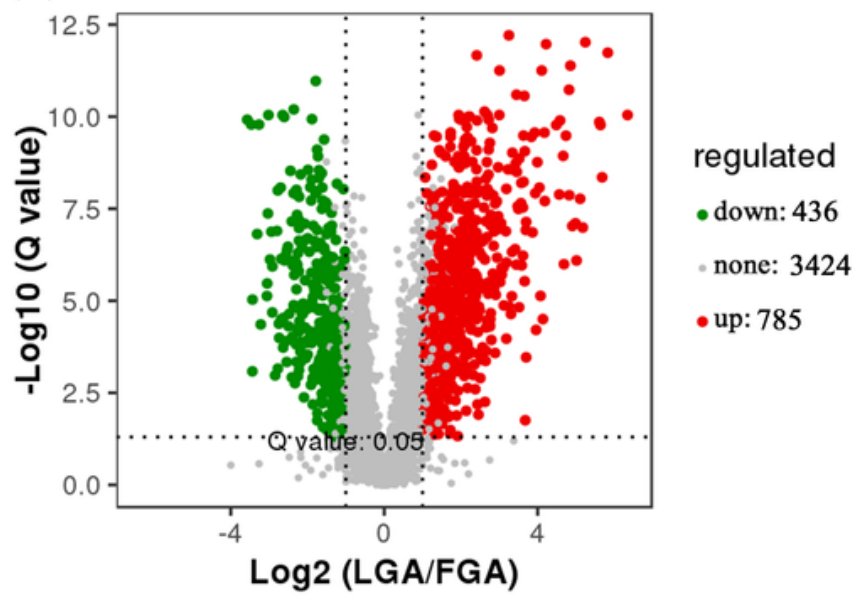

Figure 4

Volcano plot of DEGs in different comparison groups. The x-axis represents the log fold change, and the $y$-axis represents significance (q-value); green dots represent downregulated genes, and red dots represent upregulated genes. 

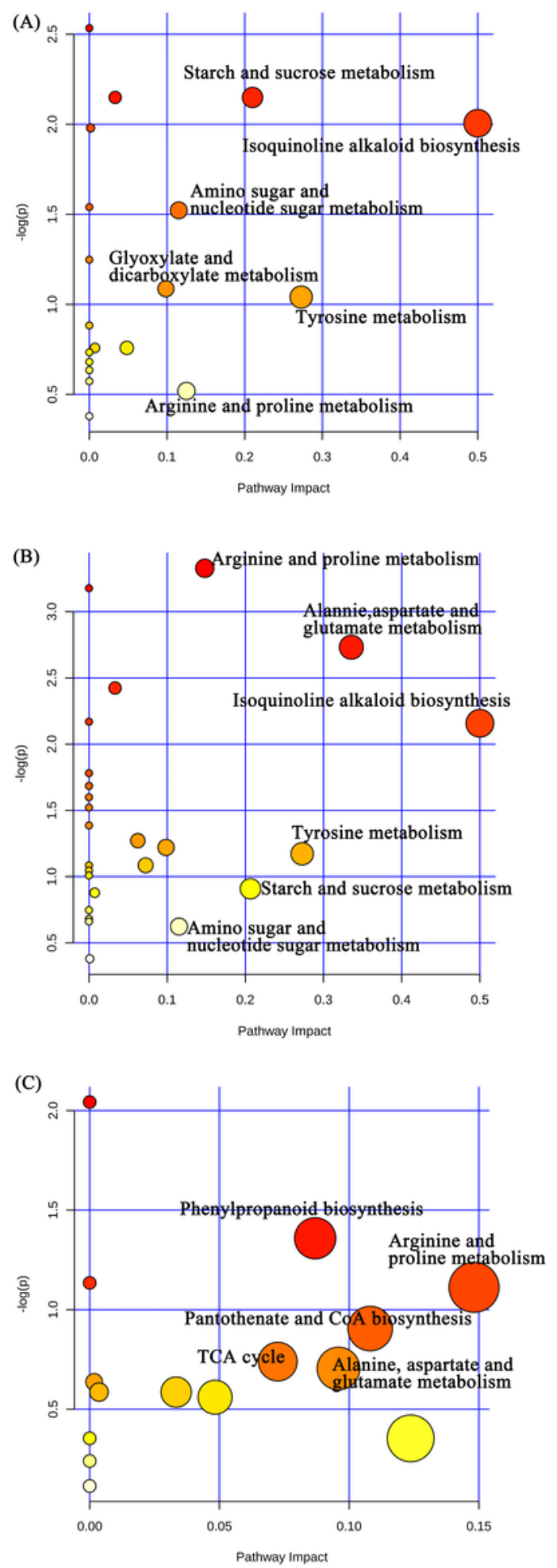

\section{Figure 5}

Pathway enrichment analysis of differentially abundant metabolites in FGA vs FS (A), LGA vs LS (B) and LGA vs FGA (C). The size and colour of the bubbles represent the pathway impact and $P$ value $(-\log (p))$ of the enrichment analysis, respectively; the darker the colour is, the more significant the enrichment. 


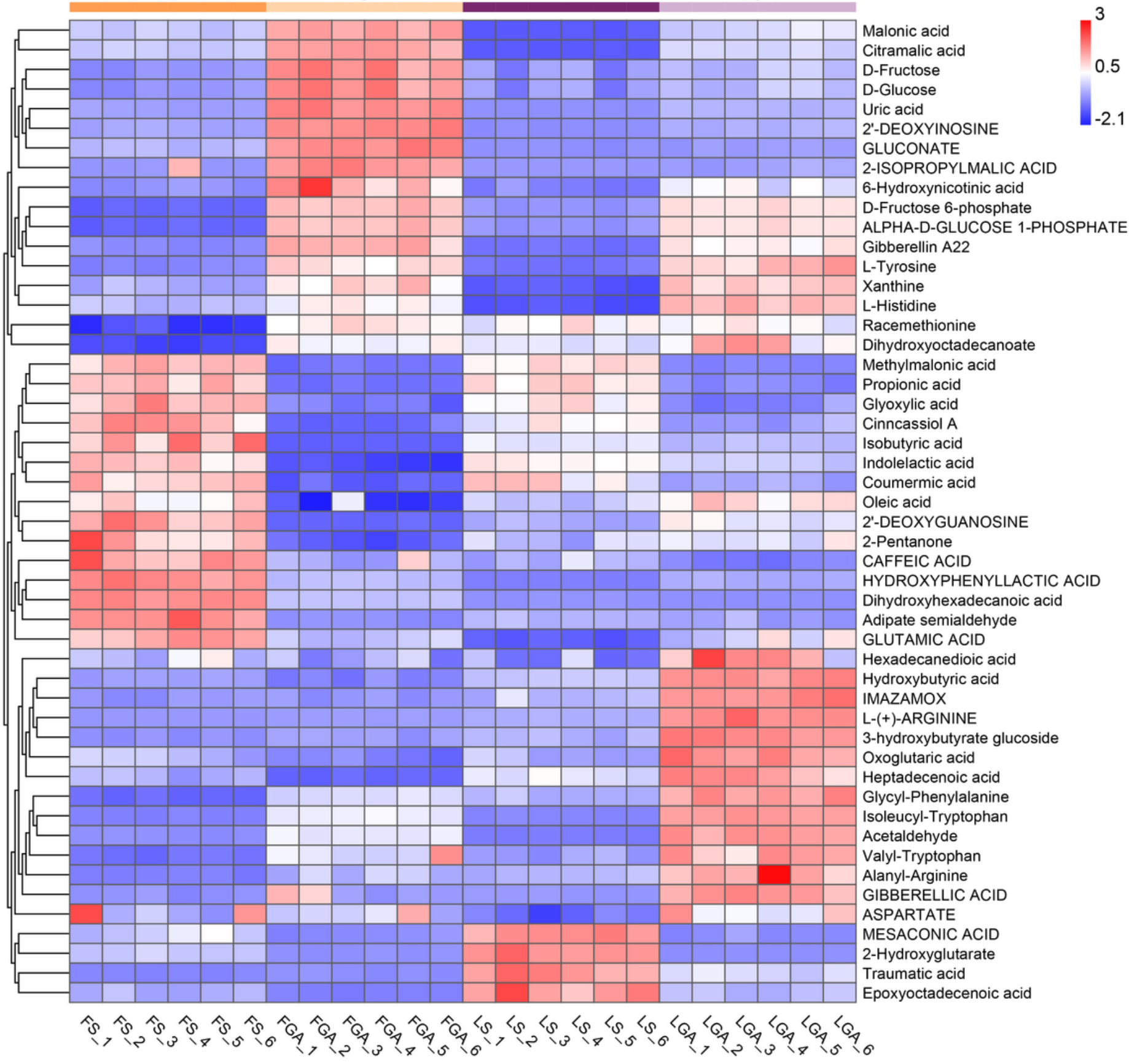

\section{Figure 6}

Clustering heatmap analysis of the main differentially abundant metabolites in the FS, FGA, LS and LGA treatments. Red indicates a higher abundance, and blue indicates a lower abundance. 


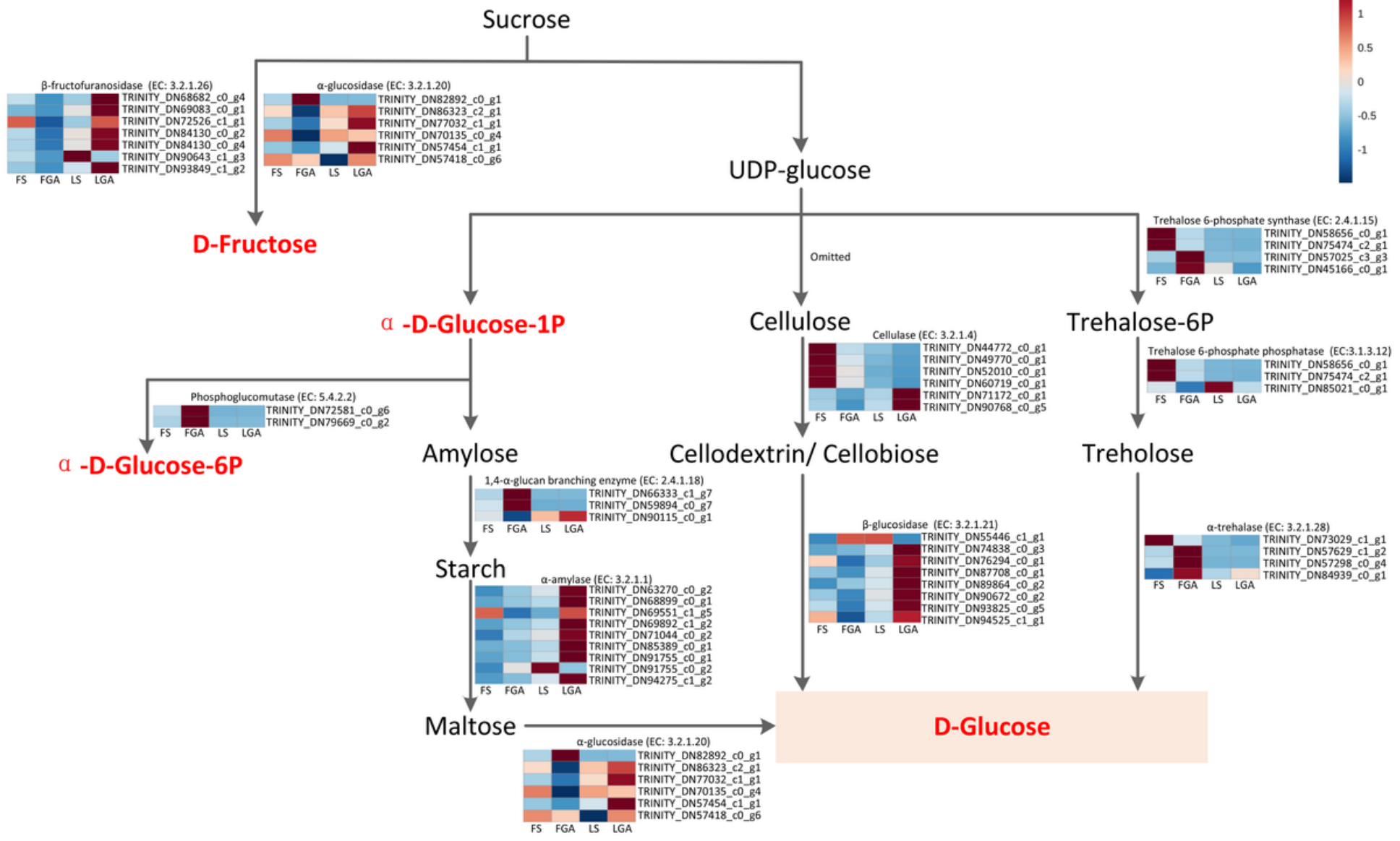

\section{Figure 7}

Starch and sucrose pathway analysis. The metabolic pathway was based on the KEGG database. Significantly differentially accumulated metabolites are indicated in bold and red, and the heatmap represents the gene expression levels in the four groups. 


\section{Genes}

AMY1.1 个

AMY1.2 个

AMY1.6 个

Exogenous GA H $_{3} \longrightarrow$

$\begin{array}{lcl}\text { GLIP5 } & \uparrow \\ \text { Cel61a } & \downarrow \\ \text { eglD } & \downarrow & \\ \text { cel1 } & \downarrow \\ \text { tpsA } & \downarrow & \\ \text { SPAC2E11.16c } & \downarrow \\ \text { TPP2 } & \downarrow & \end{array}$

\section{Metabolites}

D-glucose $\uparrow$

Maltose $\uparrow$

Cellodextrin

/Cellobiose

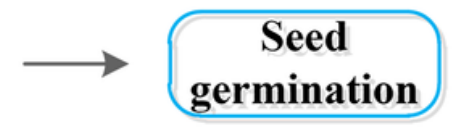

\section{Figure 8}

Diagram summarizing the mechanism of GA3-mediated disruption of seed dormancy. Genes and metabolites written in red and green were significantly upregulated and downregulated, respectively.

\section{Supplementary Files}

This is a list of supplementary files associated with this preprint. Click to download.

- TableS7.xlsx

- TableS6.xIsx

- TableS5.docx

- Tables4.docx

- TableS3.xIsx

- Tables2.xlsx

- TableS1.xIsx

- Fig.S2.tif

- Fig.S1.tif 